\title{
Inhibitor-Loaded Conducting Polymer Capsules for Active Corrosion Protection of
}

\section{Coating Defects}

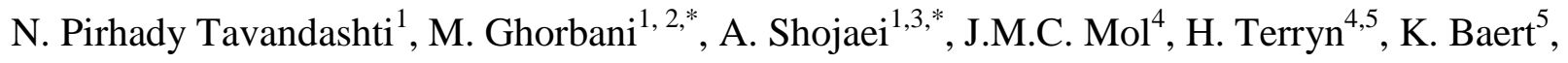 \\ Y. Gonzalez-Garcia ${ }^{4}$
}

\begin{abstract}
${ }^{1}$ Institute for Nanoscience and Nanotechnology (INST), Sharif University of Technology, P.O. Box 11155-8639, Tehran, Iran.
${ }^{2}$ Department of Materials Science and Engineering, Sharif University of Technology, P.O. Box 11155-9466, Tehran, Iran.

${ }^{3}$ Department of Chemical and Petroleum Engineering, Sharif University of Technology, PO Box 11155-9465, Tehran, Iran.

${ }^{4}$ Department of Materials Science and Engineering, Delft University of Technology, 2628CD, Delft, The Netherlands.

${ }^{5}$ Group of Electrochemical and Surface Engineering, Vrije Universiteit Brussel, B-1050, Brussels, Belgium.
\end{abstract}

\begin{abstract}
This work presents the synthesis of nanostructured hollow polyaniline (PANI) capsules and their application for encapsulation of corrosion inhibitor 2-Mercaptobenzothiazole (MBT). The $\mathrm{pH}-$ triggered release of MBT from the capsules was evidenced using UV-vis and Surface Enhanced Raman Spectroscopy (SERS). Incorporation of MBT loaded PANI capsules into epoxy ester coating on AA2024-T3 resulted in a protective system with self-healing ability confirmed by Electrochemical Impedance Spectroscopy (EIS) and Scanning Vibrating Electrode Technique (SVET). The results showed that the incorporation of MBT-loaded PANI capsules into the coating has significantly improved its corrosion protection performance due to the interesting smart characteristics of PANI capsules.
\end{abstract}

Key Words: A. Organic Coatings; A. Polymer; B. EIS; B. SVET; C. Corrosion Protection.

* Corresponding author at:

E-mail address: Ghorbani@sharif.edu (M. Ghorbani).

E-mail address: Akbar.shojaei@sharif.edu (A. Shojaei). 


\section{Introduction}

The serious consequences of the corrosion of metallic structures have become a problem of worldwide significance. One of the most common strategies to protect metallic substrates against corrosion is the application of protective organic coatings. The polymer coating acts as a barrier layer, preventing the contact of water and corrosive species with the metallic substrate, and providing a passive protection. However, various external factors such as temperature, UV radiation and mechanical forces may cause coating degradation and formation of pores and cracks in the coating through which water and corrosive species can diffuse and reach the metal surface. Thus, development of coating systems that provide both passive and active protection has attracted a great interest in recent years [1-6]. One of the most important strategies to achieve the active effect is to use micro/nanostructured hosting systems for encapsulation of corrosion inhibitors. The micro/nanocapsules are dispersed in polymer matrices, acting as reservoirs capable of controlled and smart release of the corrosion inhibitors, providing a self-healing effect. Different inhibitor encapsulation techniques have been reported in the literature, including B-cyclodextrin [7], sol-gel oxide nanoparticles [8, 9], zeolite [10], cellulose nanofibers [11, 12], ceramic containers [13, 14], halloysite nanotubes [15], layered double hydroxides (LDHs) [16], etc.

The corrosion process is usually accompanied by local changes of $\mathrm{pH}$ of the solution at and in the vicinity of active areas. These local changes in $\mathrm{pH}$ can be used as an intrinsic stimulus for smart functional containers to release the corrosion inhibitors in case of corrosion [1]. Hence, the rate of corrosion would be reduced significantly.

In this paper we show the $\mathrm{pH}$ triggered release of organic corrosion inhibitor 2mercaptobenzothiazole (MBT) from hollow conducting polymer (polyaniline) capsules. Conducting polymer micro/nanostructures have become an exciting area of research in recent years due to their special inherent properties. Their structure is switchable between different redox states and a change in their electronic charge is associated with an equivalent change in their ionic charge (via a doping- 
dedoping process) which requires mass transport between the polymer and electrolyte [17]. The ion transport can be controlled not only by adjusting electric potentials, but also changing solution $\mathrm{pH}$ $[18,19]$. Furthermore, their response to electro- or chemical stimuli can change their conductivity, color, mass, volume, permeability, and hydrophilicity [17, 19-22]. These characteristics make the conducting polymers interesting candidates to be used as micro/nanocontainers for encapsulation and smart release $[17,23]$.

Recent investigations [24-26] have demonstrated the potential application of PANI capsules for smart release of self-healing agents and corrosion inhibitors. Rohwerder et al [24] developed polyaniline hollow microspheres decorated with $\mathrm{Au}$ nanoparticles for encapsulation and redox responsive release of 3-Nitrosalicylic acid (3-NisA, corrosion inhibitor for zinc). The encapsulation of 3-NisA in the core of PANI capsules was performed during synthesis via miniemulsion technique. Crespy et al [25] used the same system for selective delivery of two payloads; i.e. in addition to 3-NisA encapsulated inside the containers, they loaded MBT on the gold nanoparticles decorated on the shell of the containers (based on the gold-thiol ( $\mathrm{Au}-\mathrm{S})$ interaction). They studied the release of the two species and claimed that a dual responsive release is obtained: $3-\mathrm{Nis}$ A is only released in response to $\mathrm{pH}$, and $\mathrm{MBT}$ is released in response to chemical reduction of capsule shell which leads to cleavage of the Au-S bond.

In this work hollow microspheres of PANI with a nanosized shell are used for the encapsulation of organic corrosion inhibitor 2-mercaptobenzothiazole (MBT). A simple encapsulation procedure was used for loading the corrosion inhibitor inside PANI capsules. The $\mathrm{pH}$ triggered release of MBT from the capsules was investigated by means of UV-vis and Surface Enhanced Raman Spectroscopy (SERS). Furthermore, the capsules were incorporated into polymer coatings applied on AA2024-T3 substrates. The self-healing corrosion protection performance of the coated samples was investigated by Electrochemical Impedance Spectroscopy (EIS) and Scanning Vibrating Electrode Technique (SVET). 


\section{Experimental Procedure}

\subsection{Materials}

Aniline ( $\geq 99.0 \%$ ), ammonium persulfate (APS, $\geq 98.0 \%$ ), sodium hydroxide ( $\geq 98.0 \%$, Pellets), hydrochloric acid (37\%), and Toluene (99.5\%) were purchased from Merck Chemicals. $\beta$ naphthalene sulfonic acid $(\beta$-NSA, $\geq 90.0 \%)$ was purchased from Fluka Chemicals, and 2Mercaptobenzothiazole (MBT) (97.0\%) was acquired from Sigma-Aldrich. All the chemicals were used as received.

\subsection{Synthesis of Nanostructured Polyaniline Capsules}

Hollow microspheres of polyaniline (PANI) with nanosized shell were synthesized using a soft template method according to a procedure we reported earlier [27]: surfactant $\beta$-naphthalene sulfonic acid ( $\beta$-NSA) was dissolved in DI water by about 10 min stirring and sonication, and then aniline monomer (mole ratio of $\beta$-NSA/aniline was 1:1) was added to the solution. The mixture was severely stirred for $15 \mathrm{~min}$, followed by $30 \mathrm{~min}$ sonication at $2-5^{\circ} \mathrm{C}$ to obtain a uniform solution. A pre-cooled aqueous solution of APS was then added. The mole ratio of APS/aniline was 1:1. The resulting solution was stirred for another $0.5 \mathrm{~min}$ to ensure complete mixing and then the reaction was left for $24 \mathrm{~h}$ to proceed at $2-5^{\circ} \mathrm{C}$ without any agitation. The resulting PANI precipitate was washed with water and methanol for several times, and was finally dried over $24 \mathrm{~h}$ at room temperature.

\subsection{Encapsulation of Corrosion Inhibitor}

First, $10 \mathrm{~mL}$ of 2-Mercaptobenzothiazole (MBT) solution with a concentration of $30 \mathrm{mg} \mathrm{mL}^{-1}$ in ethanol was prepared. Then, $50 \mathrm{mg}$ of hollow PANI microspheres was added to the solution. The mixture was stirred at $500 \mathrm{rpm}$ for $3 \mathrm{~h}$. Subsequently, the round-bottom flask containing the solution was evacuated using a vacuum pump, along with stirring for $10 \mathrm{~min}$ in order to increase the amount of encapsulation. The encapsulation procedure was followed by washing and centrifugation 
of the capsules for 3 times. The supernatant was removed and the capsules were dried at room temperature overnight.

The loading of MBT in the capsules was studied by UV-Visible Spectroscopy (PerkinElmer Lambda 950 instrument). The absorption at a wavelength of $324 \mathrm{~nm}$ was monitored, which corresponds to the absorbance peak of MBT in ethanol. The inhibitor-loaded PANI capsules were dispersed in ethanol. The dispersion was sonicated (UP400S, Hielscher Ultrasound Technology) for $20 \mathrm{~min}$, and after centrifugation $(6000 \mathrm{rpm}, 30 \mathrm{~min})$ of the solution, the supernatant was studied via UV-vis spectroscopy. As a reference, ethanol solutions of MBT with known concentrations were prepared and the calibration curve was made. The quantity of MBT loaded into the PANI capsules was $1 \mathrm{wt} \%$.

\subsection{Release of the Corrosion Inhibitor at Different pHs}

The release of MBT from the capsules was investigated by placing the capsules in dialysis bags (12 kilodalton, Sigma D0405) and introducing them into the solutions with different $\mathrm{pH}$ (i.e. $\mathrm{HCl}$ solution with $\mathrm{pH} 1.5$, DI water $\mathrm{pH} \sim 7$, and $\mathrm{NaOH}$ solution with $\mathrm{pH} 11$ ). The solutions were constantly stirred at $500 \mathrm{rpm}$ with a magnetic stirrer in order to establish equilibrium conditions. UV-vis spectroscopy was used to study the concentration of MBT in the solutions during certain periods of time. Reference solutions were made by dissolving identified concentrations of MBT in aqueous solutions with different $\mathrm{pHs}$ and the calibration curve was made for each solution. The experiments were repeated for 3 times to verify the reproducibility of the results.

\subsection{Characterization of the PANI Capsules}

The morphologies of polyaniline capsules were investigated by Scanning Electron Microscope (SEM, Zeiss Sigma VP) and a Transmission Electron Microscope (TEM, Philips EM 208). The electronic structure of PANI was studied via UV-visible spectroscopy of the dissolved polymer in 
$N$-methylpyrrolidinone (NMP) measured by a PerkinElmer Lambda 950 instrument in the range between 200 and $1000 \mathrm{~nm}$. A Fourier Transform Infrared (FTIR) spectrum of the polymer was recorded in the range of 400-4000 $\mathrm{cm}^{-1}$ with an $\mathrm{ABB}$ Bomem MB-100 FTIR spectrometer. The samples were prepared in a pellet form with spectroscopic-grade $\mathrm{KBr}$.

Surface Enhanced Raman Spectroscopy (SERS) and Raman spectroscopy were used as complementary techniques for investigation of encapsulation and release of MBT in PANI capsules. The SER and Raman spectra at 532 nm were measured with a Horiba's LabRam HR highresolution spectrometer equipped with a multichannel detection system in backscattering configuration. Laser powers were adjusted, and the acquisition times were extended in all cases to ensure an adequate signal-to-noise ratio. The spectra were obtained by using a $50 \times$ objective, and the spectral resolution of the spectrometer was about $0.5 \mathrm{~cm}^{-1}$. The SERS probe (developed inhouse at the Vrije Universiteit Brussel) consisted of silver particles $(50-500 \mathrm{~nm})$ in a gelatin matrix on a glass substrate ensuring good SERS enhancement.

\subsection{Preparation of Organic Coating Containing PANI Capsules}

Aluminum alloy AA2024-T3 was used as the base metallic substrate for corrosion protection studies. The actual chemical composition of the substrates (in wt \%) according to spark emission spectroscopy (SpectroLab, Spectro Analytical Instruments) was 4.67 Cu, 1.44 Mg, 0.55 Mn, 0.28 Si, 0.45 Fe, 0.02 Ti, 0.12 Zn, 0.01 Cr, 0.0017 Zr, 0.01 Ni, 0.01 Sb, 0.002 Sn, and 0.0014 V. To prepare the substrates for coating application, they were ground up to 1200 grid paper, washed with detergent and distilled water, and degreased in acetone in an ultrasound bath for $10 \mathrm{~min}$. Epoxy ester resin (EE430S, Rezitan) was selected for coating preparation, due to having good chemical resistance, as well as the air drying possibility and no need for curing agents which may have considerable effect on the deprotonation of polyaniline [28]. To investigate the active corrosion protection performance, AA2024 substrates were coated with different coating systems: (1) pure epoxy ester coating (control), (2) MBT directly introduced in the coating (Direct-MBT), and epoxy 
ester coating containing (3) empty PANI capsules (Empty-Caps), and (4) MBT-loaded PANI capsules (MBT@Caps).

Empty or MBT-loaded polyaniline capsules were dispersed in toluene and introduced into epoxy ester resin via sonication for 10 min to obtain a homogeneous suspension. The mixture was then applied by use of a Metrohm Autolab spin-coater (at $500 \mathrm{rpm}$ for $10 \mathrm{~s}$, then at $2000 \mathrm{rpm}$ for $20 \mathrm{~s}$ ) on AA2024 substrates. The mass loss on curing of the samples, calculated by weight, was about $60 \%$. Using this value, the final compositions of different coating systems in dried coating after curing was estimated in table 1 . The dry film thickness of the coatings was $13.9 \pm 1.1 \mu$ measured by eddy current method (ED10 Eddy, Dual Scope MP40, Germany).

\subsection{Evaluation of the corrosion protection}

In a typical EIS experiment, the coated substrates were scratched (scratch length $\approx 1 \mathrm{~mm}$ ) and placed into home-made cells, whereby an area of the sample with $8 \mathrm{~mm}$ diameter was exposed to $0.3 \mathrm{wt} \% \mathrm{NaCl}$ solution at room temperature for several days. A three-electrode set-up in a Faraday cage was used and the impedance spectra were recorded at the open circuit potential. $\mathrm{An} \mathrm{Ag} / \mathrm{AgCl}$ reference and a platinum counter electrode were immersed in the cell, and the metal substrate functioning as the working electrode, was connected to an Impedance Analyzer (Solartron S1 1287). The current response was detected in the frequency range $100 \mathrm{kHz}$ to $0.01 \mathrm{~Hz}$, and the applied voltage perturbation was $10 \mathrm{mV}$. 10 frequencies were typically assessed per decade. The EIS experiments were repeated 3 times for each coating system and the reproducibility of the data was proved. The spectra were obtained using the Zplot software and fitted with Zview. The surfaces of the scratched coatings after exposure to corrosive media were studied by JEOL JSM-IT300 SEM.

The scanning vibrating electrode technique (SVET, Applicable Electronics (USA)) was used to analyze the active anticorrosion properties of the coatings in $0.3 \mathrm{wt} \% \mathrm{NaCl}$ solution. The coating was scratched (scratch length $\approx 1 \mathrm{~mm}$ and width $\approx 25 \mu \mathrm{m}$ ) before the measurement to guarantee exposure of the metal to the aggressive solution. The coated samples were then sealed with adhesive 
tape and only the area $\left(\approx 3 \times 3 \mathrm{~mm}^{2}\right)$ including the scratch was left uncovered. Measurements on areas with dimensions of $\sim 1 \times 2 \mathrm{~mm}^{2}$ were performed every $2 \mathrm{~h}$ during $24 \mathrm{~h}$, and the mean acquisition time per scan was $\sim 20 \mathrm{~min}$. The vibrating probe was a thin Pt needle, with a platinum black sphere of ca. $20 \mu \mathrm{m}$ in diameter deposited on its tip. The current density at ca. $100 \mu \mathrm{m}$ above the coated surface was detected. The subsequent analysis of the SVET data was performed with the help of Quikgrid software. The repetition of SVET experiments showed a consistent trend in the results.

Total anodic ( $\left.\mathrm{I}_{\text {anodic }}\right)$ and cathodic $\left(\mathrm{I}_{\text {cathodic }}\right)$ currents from SVET measurements were calculated by integrating the current density distribution $\left(\mathrm{i}_{\mathrm{z}}\right)$ across the area of each scan at the time of the measurement; Equations (1) and (2):

$I_{\text {cathodic }}=\int_{x_{\min }}^{x_{\max }} \int_{y_{\min }}^{y_{\max }}\left[i_{z}(x ; y)<0\right] d x d y$

$I_{\text {anodic }}=\int_{x_{\min }}^{x_{\max }} \int_{y_{\min }}^{y_{\max }}\left[i_{z}(x ; y)>0\right] d x d y$

Where the $\mathrm{x}_{\max }, \mathrm{x}_{\min }, \mathrm{y}_{\max }$ and $\mathrm{y}_{\min }$ are the coordinates of the scanned area of each sample [29].

Salt spray tests were conducted by exposing the samples for 7 months to a salt fog atmosphere generated by spraying $5 \mathrm{wt} \%$ aqueous $\mathrm{NaCl}$ solution at $35 \pm 1.7 \circ \mathrm{C}$ in accordance with ASTMB117 specifications.

\section{Results and Discussions}

\subsection{Hollow Nanostructured Polyaniline Capsules}

Polyaniline (PANI) is one of the most promising among other conducting polymers because of its good processability, excellent environmental stability, low cost, and unique properties controllable by oxidation and protonation states [27]. PANI can be found in three well defined oxidation states: leucoemeraldine, emeraldine and pernigraniline (Scheme 1). Leucoemeraldine is the fully reduced 
(all the nitrogen atoms are amine), and pernigraniline is the fully oxidized (all the nitrogen atoms are imine) forms of polyaniline. The most stable form of PANI is emeraldine, in which the polymer chain contains equal number of oxidized and reduced units (i.e. the amine to imine ratio is approximately 1:1). The polymer can reversibly pass through different oxidation states via chemical or electrochemical oxidation and reduction. In addition to this behavior, a change in its protonation state is obtained by treatment of PANI in acidic or alkaline media. When exposed to acidic environment, PANI is protonated. Hence, anionic doping agents are introduced into the polymer chain to compensate its positive charge. Nevertheless, exposure to alkaline solution results in deprotonation of polyaniline and removing of the anionic dopant (dedoping). Therefore, PANI can exist in a minimum of six forms differing in both degree of oxidation and protonation state (Scheme 1) $[30,31]$.

Figure 1 shows the SEM image of nanostructured hollow polyaniline microspheres synthesized via a soft template chemical route [27]. It is observed that the diameter of the hollow microspheres is mostly in the range of 1-2 $\mu \mathrm{m}$. The TEM images in our previous work [27] have shown that the hollow microspheres have an average shell thickness of ca. $90 \mathrm{~nm}$. The FTIR and UV-vis spectroscopies (Figure 2) show that the capsules have a backbone polymer structure of polyaniline. Absorption peaks around 1136, 1297, 1494, and $1583 \mathrm{~cm}^{-1}$ in the FTIR spectrum of the capsules (Figure 2a) are attributed to the $\mathrm{C}=\mathrm{N}$ stretching [32, 33], $\mathrm{C}-\mathrm{N}$ stretching [33], and $\mathrm{C}=\mathrm{C}$ stretching in the benzenoid and quinoid rings [34], respectively. Meanwhile, the bands at 1035 and $508 \mathrm{~cm}^{-1}$ are related to the absorption of the $-\mathrm{SO}_{3} \mathrm{H}$ group, which directly proves that the PANI is doped with $\beta$ NSA [35]. The strong absorption peaks at 324 and $634 \mathrm{~nm}$ in the UV-vis spectrum of PANI capsules dissolved in NMP (Figure 2b) are also assigned to benzenoid and quinoid rings, respectively $[32,36]$, which confirms that the polyaniline capsules are in the emeraldine salt form. 


\subsection{Encapsulation of MBT}

The encapsulation of organic corrosion inhibitor MBT in PANI capsules was performed via stirring of a mixture of PANI and MBT in ethanol solution. UV-vis spectroscopy was used to study the encapsulation of MBT in PANI containers. The absorption at a wavelength of $324 \mathrm{~nm}$ was monitored, which corresponds to the absorbance peak of MBT in ethanol (see reference spectrum in Figure 3). After the encapsulation procedure the capsules were washed and centrifuged for several times, until no presence of MBT was observed in the supernatant of washing of the capsules (Figure 3). This was to eliminate the absorbed MBT on the surfaces of the capsules. To investigate the presence of MBT inside the polyaniline capsules, the capsules were broken by severe ultrasonication for $20 \mathrm{~min}$, and after centrifugation of the solution, the supernatant of broken capsules was studied by UV-vis spectroscopy (Figure 3). A strong absorption peak is observed in $324 \mathrm{~nm}$, which confirms that MBT was successfully loaded in PANI capsules. To verify the effect of hollow microspherical morphology of capsules and the encapsulation of MBT into the inner cavity of PANI capsules the same encapsulation procedure was applied for nanofibers of polyaniline with the same chemical structure as the hollow microspheres [27]. As shown in Figure 3 the spectrum related to nanofibers does not show any significant concentration of MBT in the supernatant after sonotrode treatment and centrifugation. It confirms that MBT was encapsulated into the inner volume of polyaniline hollow capsules.

To further study the encapsulation of MBT in PANI containers, we used micro Raman spectroscopy with applying $0.019 \mathrm{~mW}$ laser power to single capsules. Low laser powers were used to avoid photo-damage of the polymer capsules. Figure 4a shows that Raman Spectroscopy was not able to distinguish between the spectra of empty and MBT loaded PANI capsules, because the MBT concentration in the single capsules was lower than the detection limit of Raman Spectroscopy. Therefore, Surface Enhanced Raman Spectroscopy (SERS) was used to overcome the insufficient sensitivity of Raman scattering. The high sensitivity of SERS technique is mainly because of the 
electromagnetic enhancement provided by the nanostructured metal surfaces [37] although a part of the total enhancement is also attributed to the charge transfer between the chemisorbed species and the metal surface [38].

The capsules were dispersed in water and a drop of dispersion was put on the SERS probe. A silver SERS probe known for the strong enhancement of the Raman signal of MBT was used. First, 0.25 $\mathrm{mW}$ laser power was applied to the capsules. The spectra of the hollow and MBT loaded capsules did not show any difference (Figure $4 \mathrm{~b}$ ). Then, the laser power was increased to $2.5 \mathrm{~mW}$, which caused the capsules to burn off. Interestingly, the characteristic SERS signals for MBT were evidenced in the resulting spectrum (Figure $4 b$ ). It shows that after the capsules were burnt out, MBT was released and it was absorbed on the surface of the SERS probe. Due to the surface enhancement effect of SERS probe which makes it up to $10^{6}$ times more sensitive than Raman Spectroscopy [39], the MBT encapsulated in single PANI capsules was detectable. This result also confirms the encapsulation of MBT in the cavity of the capsules.

\section{3. pH Responsive Release of MBT from Hollow PANI Capsules}

The release of MBT from the capsules was investigated by placing the capsules in dialysis bags and introducing them into the solutions with different $\mathrm{pH}$ (i.e. 1.5, 7, 11). UV-vis spectroscopy was used to study the concentration of MBT in the solutions during certain periods of time. Figure 5 shows the release profile of MBT in the different $\mathrm{pH}$ environments. It is observed that the release of MBT from PANI capsules is triggered at $\mathrm{pH} 11$, and at water and acidic $\mathrm{pH}$ polyaniline capsules presented a very good suppression of MBT release saving almost all of the initial MBT inside the inner cavity.

The $\mathrm{pH}$ responsive release was further investigated by SERS. For this purpose, $0.03 \mathrm{~g}$ of the capsules was introduced in $1 \mathrm{~mL}$ of solutions with different $\mathrm{pHs}$, and after 10-15 min a drop of the solution was put on a SERS probe. If the MBT is released from the capsules, it is expected to be absorbed and measured at the probe. Therefore, the surface of SERS probe nearby the capsules was 
studied. The spectra of the samples at different pHs is shown in Figure 6. Comparing these spectra with the characteristic SERS signals of MBT (reference spectrum) shows that the only evidence for release of MBT from the PANI capsules was at $\mathrm{pH} 11$, revealing that the release is triggered quite fast in alkaline solution.

From these results can be concluded that encapsulation of MBT corrosion inhibitor in polyaniline hollow microspheres is an effective method to achieve $\mathrm{pH}$ controlled release of the inhibitor as well as to prevent its undesirable leaching from the coating. The schematic representation of the mechanism of $\mathrm{pH}$ responsive release of MBT from polyaniline capsules is shown in Figure 7. It is well known that the ion transport in conducting polymers can be controlled by changing the $\mathrm{pH}$ of the solution $[18,19,30]$. When exposed to $\mathrm{pH}<7$, PANI exists in emeraldine salt form which requires the presence of negatively charged counter ions inside the polymer chains to compensate the positive charge of the protonated polymer structure. In the emeraldine salt form of polyaniline the presence of charge compensating anions (mainly B-NSA anions) in the polymer structure produces an osmotic pressure, that forces additional water to enter the polymer phase [40-42]. The hydrophilicity of the polymer is also increased [43, 44]. However, when the polyaniline capsules are exposed to the solution with $\mathrm{pH}>>7$, the polymer chains are deprotonated. Therefore, to keep the overall charge neutrality, ß-NSA dopant anions are expelled towards the solution. The dedoping effect at higher $\mathrm{pH}$ is accompanied by reduction of the hydrophilicity of the polymer. Therefore, the possibility of permeation of hydrophobic MBT molecules through the PANI shell is increased. Moreover, at $\mathrm{pH} \gg>7$ the exit of $\mathrm{B}$-NSA dopant anions from the polymer structure may result in contraction of the capsules. Polyaniline shell contraction produces a hydrodynamic force inside the capsules, which facilitates expulsion of MBT molecules through openings or cracks at the surface of the microspheres [17]. 


\subsection{Evaluation of the Self-healing Performance}

During corrosion in neutral aqueous solution an increase in the local $\mathrm{pH}$ can occur through reduction of the dissolved oxygen (1) [45, 46]:

$\mathrm{O}_{2}+2 \mathrm{H}_{2} \mathrm{O}+4 \mathrm{e}^{-} \rightarrow 4 \mathrm{OH}^{-}$

This increase of $\mathrm{pH}$ is the trigger for MBT-loaded PANI capsules incorporated in the coating to release the corrosion inhibitor. MBT in itself is an effective inhibitor for corrosion of aluminum alloys $[1,16]$, which forms a protective layer on the attacked metal surface.

In this section the corrosion inhibiting performance of the MBT loaded PANI capsules when incorporated into the epoxy ester coating is studied. Empty and MBT-loaded capsules were incorporated in an epoxy ester coating and the corrosion performance was compared to that of the pure epoxy ester coating (control sample) and a sample containing MBT mixed directly into the coating. The compositions of the different coating systems and codes used during the investigation are summarized in Table 1.

\subsubsection{EIS Measurements}

To study the self-healing properties of the coatings EIS corrosion tests were performed on the scratched samples in $0.3 \mathrm{wt} \% \mathrm{NaCl}$ solution. Selected Bode plots of the scratched samples after 1 and 7 days immersion are shown in Figure 8 and Figure 9, respectively. Figure 8 shows that the impedance values of the coating containing MBT-loaded PANI capsules (MBT@Caps) were higher than that of the other coating systems after 1 day of immersion, and the impedance values of Empty-Caps, Direct-MBT and control coating systems were almost similar to each other. Figure 9 shows that MBT@Caps coating after 7 days immersion still has the highest impedance values amongst other coating systems. Its superior corrosion protection compared to other samples is more pronounced after 7 days, and the low frequency impedance of MBT@Caps coating is one order of magnitude higher than that of the control sample. The low frequency impedance of the Empty-Caps 
and Direct-MBT coating systems were approximately similar to each other and higher than that of the control sample after 7 days immersion.

Generally, three time constants were observed in different coatings during immersion in $\mathrm{NaCl}$ solution. The time constant at high frequencies $\left(\sim 10^{5} \mathrm{~Hz}\right)$ is ascribed to the capacitance of the coating. The time constant at medium frequency range can be assigned to an oxide layer [8], which may be composed of natural aluminum oxide or the passive oxide layer that forms on the surface due to interaction of PANI or MBT with the attacked metal surface. The third time constant detected in the low frequency range may correspond to the double layer capacitance of the corrosion areas. Meanwhile, each mentioned capacitive response was associated with a corresponding resistive response. Accordingly, the interpretation of impedance spectra of different samples was performed after numerical fitting using the equivalent circuit presented in Figure 10d. In this equivalent circuit $\mathrm{R}_{\text {sol }}$ is the solution resistance; $\mathrm{R}_{\text {coat }}$ and $C P E_{\text {coat }}$ are the resistance and capacitance of the coating, respectively; $\mathrm{R}_{\text {oxide }}$ is the resistance of oxide layer and $\mathrm{CPE}_{\text {oxide }}$ is the capacitance of oxide layer; $\mathrm{R}_{\mathrm{p}}$ and $\mathrm{CPE}_{\mathrm{dl}}$ are the polarization resistance and double-layer capacitance, respectively. Constant phase elements (CPE) were used in all fittings (Figure 10d) instead of capacitances because of the deviation from the ideal capacitance behavior (the phase angle of capacitors was different from $-90^{\circ}$ ). The impedance of a CPE is defined as:

$Z_{\mathrm{CPE}}(\omega)=Q^{-1}(j \omega)^{-n}$

where $\mathrm{Z}$ is the impedance, $\mathrm{Q}$ is the admittance coefficient, $\mathrm{j}^{2}=-1$ is the imaginary number, $\omega$ is the angular frequency $\left(\operatorname{rad~s} s^{-1}\right.$ ), and $\mathrm{n}$ is the CPE exponent. The following equation was used to calculate capacitance values for the different elements in the equivalent circuit:

$\mathrm{C}=\mathrm{Q}\left(\omega_{\max }\right)^{\mathrm{n}-1}$

where $\omega_{\max }$ is the frequency at which the imaginary impedance reaches a maximum for the respective time constant [47]. 
Figure 10a shows the evolution of the capacitance for the oxide layer in different scratched coatings during immersion in $0.3 \mathrm{wt} \% \mathrm{NaCl}$ solution. It is observed that the coating systems containing empty or MBT loaded PANI capsules show stable capacitance values during 7 days of immersion. The capacitance of these coatings has approximately similar values during the whole immersion period. Initially, the capacitance $\left(\mathrm{C}_{\text {oxide }}\right)$ of Direct-MBT coating system was also close to the coatings containing polyaniline, but after 5 days it showed a significant increase. However, the largest capacitance values among all coating systems and throughout the entire immersion period were measured for the control sample, which has a substantial increasing trend after 3 days. It indicates degradation in the oxide layer of the control coating with time. It also evidences that a more compact oxide layer is formed in the coatings containing PANI capsules.

The compactness of the oxide layer formed on the aluminum substrate is very important from the point of view of corrosion protection, since it can prevent direct ingression of corrosive species to the bare metallic surface [8]. The evolution of the resistance of the oxide layer in different coating systems during immersion in $\mathrm{NaCl}$ solution is presented in Figure 10b. The control coating (pure epoxy ester coating) shows the smallest values of $\mathrm{R}_{\text {oxide }}$ among other coating systems. This is in agreement with the highest values of capacitance of oxide layer observed for this system, showing that the pure epoxy ester coating has the weakest oxide layer among other systems. The resistance values of oxide layer of the Direct-MBT coating was only slightly higher than that of the control sample, which can show that direct incorporation of MBT in epoxy ester coatings was not a successful way to increase the corrosion protection performance. It may be due to the strong interaction of MBT with epoxy ester matrix which may have caused inhibitor deactivation [1]. The resistance values of oxide layer for the Empty-Caps coating system is about 2-3 times higher than that of the control system during 7 days of immersion, showing that the embedding of polyaniline microspheres has improved the corrosion protection properties of the epoxy ester coatings. Nevertheless, the largest resistance values of oxide layer among all coating systems and during the entire immersion period were measured for the coating containing MBT loaded PANI capsules 
(MBT@Caps system). Interestingly, the resistance increases with immersion time from $2.3 \times 10^{6}$ (initial value) to $1.2 \times 10^{7} \Omega \mathrm{cm}^{2}$ after 7 days, and finally reaches one order of magnitude higher value than the control sample. The increase of resistance of oxide layer clearly indicates the selfhealing of the defect area in the MBT@Caps coating system.

The rate of the corrosion process can be estimated by measuring the polarization resistance. Figure 10c illustrates that the evolution of the polarization resistance for different coating systems with immersion time shows a similar trend to the resistance of the oxide layer (Figure 10b). It is observed that the control coating has the smallest values of polarization resistance among other coating systems, which means that this coating has the highest corrosion activity. However, the largest values of $R_{p}$ for the coating containing MBT loaded PANI capsules confirm superior corrosion protection properties of this coating system. The polarization resistance of MBT@Caps coating system increases with immersion time from $3.85 \times 10^{6}$ (initial value) to $1.74 \times 10^{7} \Omega \mathrm{cm}^{2}$ after 7 days which is one order of magnitude higher than that of the control sample. The values of polarization resistance in the coating containing empty PANI capsules (Empty-Caps system) were approximately similar to that of the MBT@Caps coating system only in the first 2 days of immersion. But in longer immersion times a significant reduction in the values of $R_{p}$ occurred for Empty-Caps system. It shows that loading of PANI capsules with MBT corrosion inhibitor has obviously influenced the self-healing ability and corrosion protection properties of the coating. The PANI capsules, not only capable of passivating the metallic surface but also store the corrosion inhibitor and prevent unwanted interactions between MBT and epoxy ester coating. When corrosion starts (in case of damage in the coating) the capsules release the corrosion inhibitor to stop the corrosion process, providing a self-healing effect.

In general, it is observed that the corrosion protection performance of the coating is improved by the incorporation of hollow polyaniline microspheres into the epoxy ester coating. This effect is due to the unique electrochemical properties of polyaniline and that it can undergo different oxidation 
states: leucoemeraldine, emeraldine and pernigraniline (Scheme 1). Electroactive conducting polymers have been widely used as promising candidates for corrosion protection of ferrous and non-ferrous alloys [48-53]; an explanation of the mechanism of corrosion protection of metals by polyaniline coatings is that a protective PANI induced passive oxide layer forms on the metallic surface via the cyclic reduction of emeraldine salt form of polyaniline to its leucoemeraldine form, with subsequent re-oxidation of the latter species by oxygen.

Figure 11 shows the SEM images of the scratched coating systems after 4 days of exposure to 0.3 wt\% $\mathrm{NaCl}$ solution. A good agreement is observed between these SEM results and the EIS results discussed above. Figure 11a,b demonstrates that for both control and Direct-MBT coating systems large aggregates of corrosion products are formed in the vicinity of scratch, which evidences the high corrosion activity. Moreover, presence of regions of undercoating corrosion and also delamination of the coating in some areas is detected for both coating systems. For the coating systems containing empty or MBT loaded PANI capsules (Figure 11c,d) no signs of delamination or undercoating corrosion was observed. However, formation of corrosion products in the scratch area of Empty-Caps system confirms the lower corrosion protection ability of this coating compared to MBT@Caps system.

\subsubsection{SVET Measurements}

The scanning vibrating electrode technique (SVET) is employed to study the corrosion activity at a coating defect and to evaluate the protection performance of the corrosion inhibitor. This technique monitors localized corrosion activity by mapping the distribution of cathodic and anodic ionic currents along the surface. SVET maps of the coating defect area immersed in the $0.3 \% \mathrm{NaCl}$ solution were obtained every two hours. In Figure 12 a-d the current density maps of the different coated systems for selected immersion times (immediately, 10, and $18 \mathrm{~h}$ after immersion) are shown. In Figure 13 a-d the evolution with time of the total cathodic and anodic currents from 
SVET maps is displayed. In general the anodic currents for all samples except the control sample showed very low values.

The pure epoxy ester coating showed localized corrosion activity in the scratch immediately after immersion (Figure 12a). Initially only anodic activity at both ends of the scratch is detected. With longer immersion time, also cathodic activity could be observed (see Figure 12 a after 10 and 18 hours). In general the scratched pure epoxy ester coatings presented increasing localized corrosion activity with immersion time during all tests (Figure 12a and Figure 13a).

The SVET maps for the scratched Direct-MBT coating system are shown in Figure 12b. Lower corrosion activity is observed compared to the control coating. Interestingly, an increasing cathodic activity was observed after few hours of immersion while no anodic activity was detected (Figure $12 \mathrm{~b}$ and Figure 13b). This indicated that the anodic activity is occurring underneath the coating around the defect, which cannot be detected by the SVET technique. This observation is in agreement with SEM images from Figure 11.

Similar performance was observed for the scratched coatings containing empty PANI capsules (see Figure 12c and Figure 13c). However, now the cathodic currents showed slightly lower values than for the pure epoxy ester coated samples. From SEM images delamination was not clearly detected, only certain accumulation of corrosion products at the proximities of the scratch (Figure 11c). However, the SVET results suggest that indeed after 10 hours of immersion corrosion is occurring underneath the coating. This is evidenced by the detection of cathodic currents only.

In the case of the epoxy ester coating containing MBT-loaded PANI capsules the SVET results showed a different behavior compared to the other samples (Figure $12 \mathrm{~d}$ and Figure 13d). Current density maps showed no activity during the whole period of immersion. It is observed in Figure $13 \mathrm{~d}$ that anodic and cathodic currents remained almost constant and within the noise level. This result is in agreement with the EIS observations. It confirms that the incorporation of MBT loaded polyaniline capsules improves the corrosion protection performance of the coatings. Furthermore it 
demonstrates that the MBT inhibitor indeed is released from the PANI capsules when the local $\mathrm{pH}$ in the surrounding media increases. SVET results highlight a synergistic effect between the protection ability of MBT as corrosion inhibitor and the polyaniline microspheres acting as smart additives for organic coatings.

\subsubsection{Salt-spray Test}

The coated samples were exposed to salt fog ambient for 7 months to investigate the long term corrosion protection performance (Figure 14). In conformance to electrochemical measurements the MBT@Caps coating system has shown enhanced performance of corrosion protection in the salt spray test. Undercoating corrosion is observed for the control coating; color-change and presence of white corrosion products on the surface of the substrate is clearly evident. Blistering in control coating system started from the initial months of exposure to salt fog. However, Direct-MBT and Empty-Caps coating systems show more severe signs of corrosion and presence of numerous pits is detectable on their surfaces. The first signs of deterioration among all coating systems occurred in Direct-MBT system, and it was after $1800 \mathrm{~h}$ of exposure. This result confirms that direct introduction of MBT in epoxy ester coating have negatively affected the corrosion protection performance of the coating. This can be due to interaction of coating matrix and corrosion inhibitor which may have resulted to inhibitor deactivation and/or coating degradation.

\section{Conclusions}

In the present work we synthesized and used hollow nanostructured polyaniline capsules for encapsulation of organic corrosion inhibitor 2-Mercaptobenzothiazole (MBT). The $\mathrm{pH}$ responsive release of MBT from the capsules is observed at higher pHs $(\sim 11)$, which makes them good candidates for incorporation into corrosion protective coatings. The embedding of MBT loaded PANI capsules in an epoxy ester coating on AA2024-T3 substrate allows for a corrosion inhibiting effect to be obtained upon exposure to an aqueous electrolyte. The superior corrosion protection of this active system in comparison to the pure epoxy ester coating was observed by Electrochemical 
Impedance Spectroscopy (EIS) and the Scanning Vibrating Electrode Technique (SVET). The impedance value of the scratched area of the coating containing MBT loaded PANI capsules after 7 days of immersion was one order of magnitude higher than that of the pure epoxy ester coating. The SVET results showed no signs of corrosion activity in the scratched area of this coating up to $24 \mathrm{~h}$. Additionally, results indicated an increase of the barrier properties of the coating by the presence of the polyaniline capsules. In conclusion, the viability of using polyaniline for encapsulation of MBT corrosion inhibitor is shown. The release of the inhibitor was triggered by an increase of $\mathrm{pH}$ during the initial stages of corrosion in a coating defect. Incorporation of MBT-loaded PANI capsules in the coating resulted in enhanced active protection performance in case of a coating defect without compromising the barrier properties the coating.

\section{Acknowledgments}

The authors from Sharif University of Technology would like to express the appreciation to the Iran National Science Foundation for financial support.

\section{References}

1. M.L. Zheludkevich, J. Tedim, M.G.S. Ferreira, "Smart" Coatings for Active Corrosion Protection Based on Multi-functional Micro and Nanocontainers, Electrochim. Acta 82 (2012) 314-323.

2. Y. González-García, J.M.C. Mol, T. Muselle, I. De Graeve, G. Van Assche, G. Scheltjens, B. Van Mele, H. Terryn, A Combined Mechanical, Microscopic and Local Electrochemical Evaluation of Self-healing Properties of Shape-memory Polyurethane Coatings, Electrochim. Acta 56 (2011) 9619-9626.

3. Y. González-García, S.J. García, A.E. Hughes, J.M.C. Mol, A Combined RedoxCompetition and Negative-feedback SECM Study of Self-healing Anticorrosive Coatings, Electrochem. Commun. 13 (2011) 1094-1097. 
4. Y. González-García, J.M.C. Mol, T. Muselle, I. De Graeve, G. Van Assche, G. Scheltjens, B. Van Mele, H. Terryn, SECM Study of Defect Repair in Self-healing Polymer Coatings on Metals, Electrochem. Commun. 13 (2011) 169-173.

5. A. Ghazi, E. Ghasemi, M. Mahdavian, B. Ramezanzadeh, M. Rostami, The Application of Benzimidazole and Zinc Cations Intercalated Sodium Montmorillonite as Smart Ion Exchange Inhibiting Pigments in the Epoxy Ester Coating, Corros. Sci. 94 (2015) 207217.

6. G. Williams, S. Geary, H. N. McMurray, Smart Release Corrosion Inhibitor Pigments Based on Organic Ion-Exchange Resins, Corros. Sci. 57 (2012) 139-147.

7. A.N. Khramov, N.N. Voevodin, V.N. Balbyshev, M.S. Donley, Hybrid Organo-Ceramic Corrosion Protection Coatings with Encapsulated Organic Corrosion Inhibitors, Thin Solid Films 447 (2004) 549-557.

8. M.L. Zheludkevich, R. Serra, M.F. Montemor, K.A. Yasakau, I.M. Miranda Salvado, M.G.S. Ferreira, Nanostructured Sol-gel Coatings Doped with Cerium nitrate as Pretreatments for AA2024-T3: Corrosion Protection Performance, Electrochim. Acta 51 (2005) 208-217.

9. N. Pirhady Tavandashti, S. Sanjabi, Corrosion Study of Hybrid Sol-gel Coatings Containing Boehmite Nanoparticles Loaded with Cerium nitrate Corrosion Inhibitor, Prog. Org. Coat. 69 (2010) 384-391.

10. S. Dias, S.V. Lamaka, C.A. Nogueira, T.C. Diamantino, M.G.S. Ferreira, Sol-gel Coatings Modified with Zeolite Fillers for Active Corrosion Protection of AA2024, Corros. Sci. 62 (2012) 153-162. 
11. A. Yabuki, A. Kawashima, I.W. Fathona, Self-healing Polymer Coatings with Cellulose Nanofibers Served as Pathways for the Release of a Corrosion Inhibitor, Corros. Sci. 85 (2014) 141-146.

12. A. Yabuki, T. Shiraiwa, I.W. Fathona, pH-Controlled Self-Healing Polymer Coatings with Cellulose Nanofibers Providing an Effective Release of Corrosion Inhibitor, Corros. Sci. 103 (2016) 117-123.

13. I.A. Kartsonakis, E. Athanasopoulou, D. Snihirova, B. Martins, M.A. Koklioti, M.F. Montemor, G. Kordas, C.A. Charitidis, Multifunctional Epoxy Coatings Combining a Mixture of Traps and Inhibitor Loaded Nanocontainers for Corrosion Protection of AA2024-T3, Corros. Sci. 85 (2014) 147-159.

14. I.A. Kartsonakis, A.C. Balaskas, E.P. Koumoulos, C.A. Charitidis, G.C. Kordas, Incorporation of Ceramic Nanocontainers into Epoxy Coatings for the Corrosion Protection of Hot Dip Galvanized Steel, Corros. Sci. 57 (2012) 30-41.

15. D. Fix, D.V. Andreeva, Y.M. Lvov, D.G. Shchukin, H. Mohwald, Application of Inhibitor-Loaded Halloysite Nanotubes in Active Anti-Corrosive Coatings, Adv. Funct. Mater. 19 (2009) 1720-1727.

16. J. Tedim, S.K. Poznyak, A. Kuznetsova, D. Raps, T. Hack, M.L. Zheludkevich, M.G.S. Ferreira, Enhancement of Active Corrosion protection via Combination of InhibitorLoaded Nanocontainers, Appl. Mater. Interfaces 2 (2010) 1528-1535.

17. M.R. Abidian, D.H. Kim, D.C. Martin, Conducting-Polymer Nanotubes for Controlled Drug Release, Adv. Mater. 18 (2006) 405-409.

18. A. Michalska, K. Maksymiuk, Counter-Ion Influence on Polypyrrole Potentiometric pH Sensitivity, Microchim. Acta 143 (2003) 163-175. 
19. J.H. Hwang, M. Pyo, pH-Dependent Mass and Volume Changes of Polypyrrole/Poly (styrene sulfonate), Bull. Korean Chem. Soc. 27 (2006) 2067-2070.

20. J. Huang, B. Quan, M. Liu, Z. Wei, L. Jiang, Conducting Polypyrrole Conical Nanocontainers: Formation Mechanism and Voltage Switchable Property, Macromol. Rapid Commun. 29 (2008) 1335-1340.

21. D.L. Pile, Y. Zhang, A.C. Hillier, Electrochemically Modulated Permeability of Poly (aniline) and Composite Poly (aniline)-Poly (styrenesulfonate) Membranes, Langmuir 22 (2006) 5925-5931.

22. M. Pyo, C.H. Kwak, In Situ Scanning Tunneling Microscopy Study on Volume Change of Polypyrrole/poly (styrene sulfonate), Synth. Met. 150 (2005) 133-137.

23. D. Kepinska, G.J. Blanchard, P. Krysinski, J. Stolarski, K. Kijewska, M. Mazur, PyreneLoaded Polypyrrole Microvessels, Langmuir 27 (2011) 12720-12729.

24. A. Vimalanandan, L.P. Lv, T.H. Tran, K. Landfester, D. Crespy, M. Rohwerder, Redox-Responsive Self-Healing for Corrosion Protection, Adv. Mater. 25 (2013) 69806984.

25. L.P. Lv, K. Landfester, D. Crespy, Stimuli-Selective Delivery of Two Payloads from Dual Responsive Nanocontainers, Chem. Mater. 26 (2014) 3351-3353.

26. L.P. Lv, Y. Zhao, N. Vilbrandt, M. Gallei, A. Vimalanandan, M. Rohwerder, K. Landfester, D. Crespy, Redox Responsive Release of Hydrophobic Self-Healing Agents from Polyaniline Capsules, J. Am. Chem. Soc. 135 (2013) 14198-14205.

27. N. Pirhady Tavandashti, M. Ghorbani, A. Shojaei, Controlled Growth of Hollow Polyaniline structures: from Nanotubes to Microspheres, Polym. 54 (2013) 5586-5594. 
28. R. Arefinia, A. Shojaei, H. Shariatpanahi, J. Neshati, Anticorrosion Properties of Smart Coating Based on Polyaniline Nanoparticles/Epoxy-ester System, Prog. Org. Coat. 75 (2012) 502-508.

29. M.F. Montemor, D.V. Snihirova, M.G. Taryba, S.V. Lamaka, I.A. Kartsonakis, A.C. Balaskas, M.G.S. Ferreira, Evaluation of self-healing ability in protective coatings modified with combinations of layered double hydroxides and cerium molibdate nanocontainers filled with corrosion inhibitors, Electrochim. Acta 60 (2012) 31-40.

30. I.Y. Sapurina, M.A. Shishov, Oxidative Polymerization of Aniline: Molecular Synthesis of Polyaniline and the Formation of Supramolecular Structures, INTECH Open Access Publisher, 2012.

31. N. Gospodinova, L. Terlemezyan, Conducting Polymers Prepared by Oxidative Polymerization: Polyaniline, Prog. Polym. Sci. 23 (1998) 1443-1484.

32. H. Zhang,Y. Li, X. Wang, J. Li, F. Wang, A Facile Route to Hollow Microspherical Polyaniline, Polym. 52 (2011) 4246-4252.

33. C. Luo, H. Peng, L. Zhang, G.L. Lu, Y. Wang, J. Travas-Sejdic, Formation of Nano/Microstructures of Polyaniline and its Derivatives, Macromol. 44 (2011) 6899-6907.

34. J. Li, Q. Jia, J. Zhu, M. Zheng, Interfacial Polymerization of Morphologically Modified Polyaniline: From Hollow Microspheres to Nanowires, Polym. Int. 57 (2008) 337-341.

35. Z. Wei, M. Wan, Hollow Microspheres of Polyaniline Synthesized with an Aniline Emulsion Template, Adv. Mater. 14 (2002) 1314-1317.

36. J. Han, P. Fang, J. Dai, R. Guo, One-Pot Surfactantless Route to Polyaniline Hollow Nanospheres with Incontinuous Multicavities and Application for the Removal of Lead Ions from Water, Langmuir 28 (2012) 6468-6475. 
37. M. Moskovits, Surface-Enhanced Spectroscopy, Rev. Mod. Phys. 57 (1985) 783.

38. J.R. Lombardi, R.L. Birke, A Unified Approach to Surface-Enhanced Raman Spectroscopy, J. Phys. Chem. C 112 (2008) 5605-5617.

39. E.J. Blackie, E.C.L. Ru, P.G. Etchegoin, Single-Molecule Surface-Enhanced Raman Spectroscopy of Non-Resonant Molecules, J. Am. Chem. Soc. 131 (2009) 14466-14472.

40. L. Bay, T. Jacobsen, S. Skaarup, K. West, Mechanism of Actuation in Conducting Polymers: Osmotic Expansion, J. Phys. Chem. B 105 (2001) 8492-8497.

41. H. Zengin, H.G. Spencer, G. Zengin, R.V. Gregory, Studies of Solution Properties of Polyaniline by Membrane Osmometry, Synth. Met. 157 (2007) 147-154.

42. M. Roemer, T. Kurzenknabe, E. Oesterschulze, N. Nicoloso, Microactuators Based on Conducting Polymers, Anal. Bioanal. Chem. 373 (2002) 754-757.

43. H. Deligoz, Preparation of Self-Standing Polyaniline-Based Membranes: Doping Effect on the Selective Ion Separation and Reverse Osmosis Properties, J. Appl. Polym. Sci. 105 (2007) 2640-2645.

44. G. Inzelt, M. Pineri, J.W. Schultze, M.A. Vorotyntsev, Electron and Proton Conducting Polymers: Recent Developments and /Prospects, Electrochim. Acta 45 (2000) 2403-2421.

45. A. Boag, A.E. Hughes, A.M. Glenn, T.H. Muster, D. McCulloch, Corrosion of AA2024T3 Part I: Localised Corrosion of Isolated IM Particles, Corros. Sci. 53 (2011) 17-26.

46. A.M. Glenn, T.H. Muster, C. Luo, X. Zhou, G.E. Thompson, A. Boag, A.E. Hughes, Corrosion of AA2024-T3 Part III: Propagation, Corros. Sci. 53 (2011) 40-50.

47. C.H. Hsu, F. Mansfeld, Technical Note: Concerning the Conversion of the Constant Phase Element Parameter $Y_{0}$ into a Capacitance, Corros. 57 (2001) 09. 
48. D.E. Tallman, G. Spinks, A. Dominis, G.G. Wallace, Electroactive Conducting Polymers for Corrosion Control. Part 1. A Review of Non-Ferrous Metals, J. Solid State Electrochem. 6 (2002) 73-84.

49. G. Spinks, A.J. Dominis, G.G. Wallace, D.E. Tallman, Electroactive Conducting Polymers for Corrosion Control. Part 2. Ferrous Metals, J. Solid State Electrochem. 6 (2002) 85100.

50. X. Bai, T.H. Tran, D. Yu, A. Vimalanandan, X. Hu, M. Rohwerder, Novel Conducting Polymer Based Composite Coatings for Corrosion Protection of Zinc, Corros. Sci. 95 (2015) 110-116.

51. B.D. Mert, Corrosion Protection of Aluminum by Electrochemically Synthesized Composite Organic Coating, Corros. Sci. 103 (2016) 88-94.

52. G. Gupta, N. Birbilis, A.B. Cook, A.S. Khanna, Polyaniline-Lignosulfonate/Epoxy Coating for Corrosion Protection of AA2024-T3, Corros. Sci. 67 (2013) 256-267.

53. M. Sababi, J. Pan, P.E. Augustsson, P.E. Sundell, P.M. Claesson, Influence of Polyaniline and Ceria Nanoparticle Additives on Corrosion Protection of a UV-Cure Coating on Carbon Steel, Corros. Sci. 84 (2014) 189-197. 
Table 1. Description of different coating systems.

\begin{tabular}{cc}
\hline Sample & Description \\
\hline Control & Pure epoxy ester coating \\
Direct-MBT & $0.5 \mathrm{wt} \%$ of MBT mixed directly into the coating \\
Empty-Caps & $1 \mathrm{wt} \%$ of empty PANI capsules embedded into the coating \\
MBT@Caps & $1 \mathrm{wt} \%$ of MBT loaded PANI capsules embedded into the coating \\
\hline
\end{tabular}




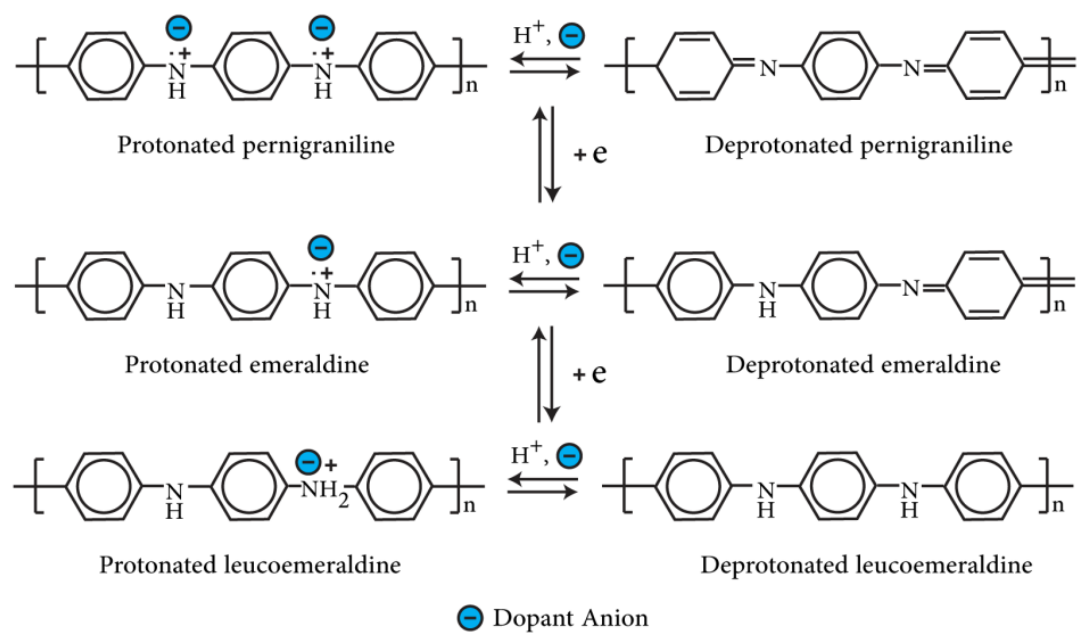

Scheme 1. Possible forms of polyaniline. 


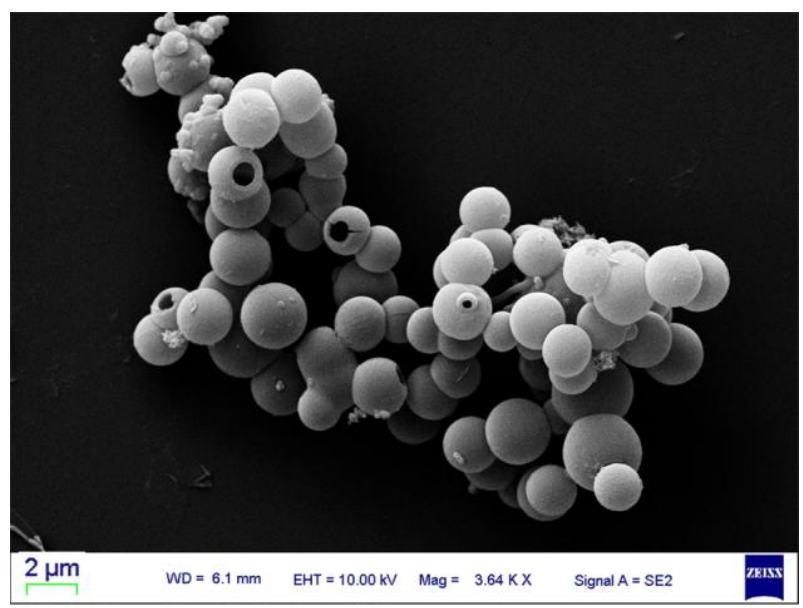

Figure 1. SEM image of hollow polyaniline capsules. 

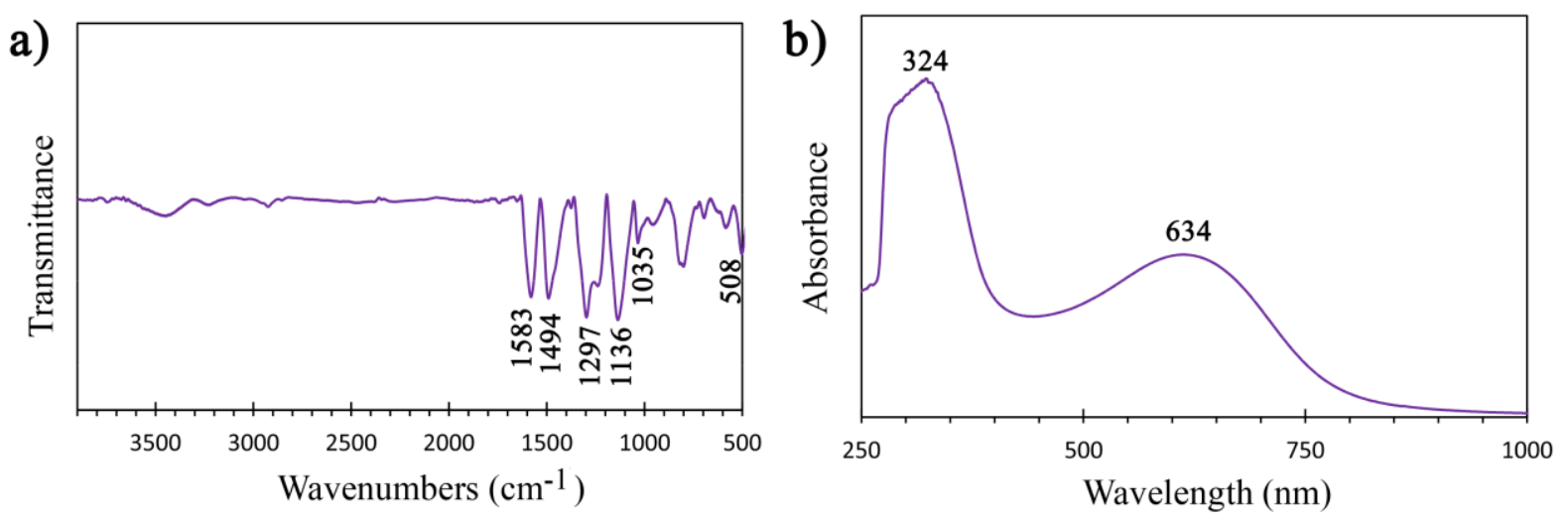

Figure 2. FTIR (a) and UV-vis (b) spectra of hollow polyaniline capsules. 


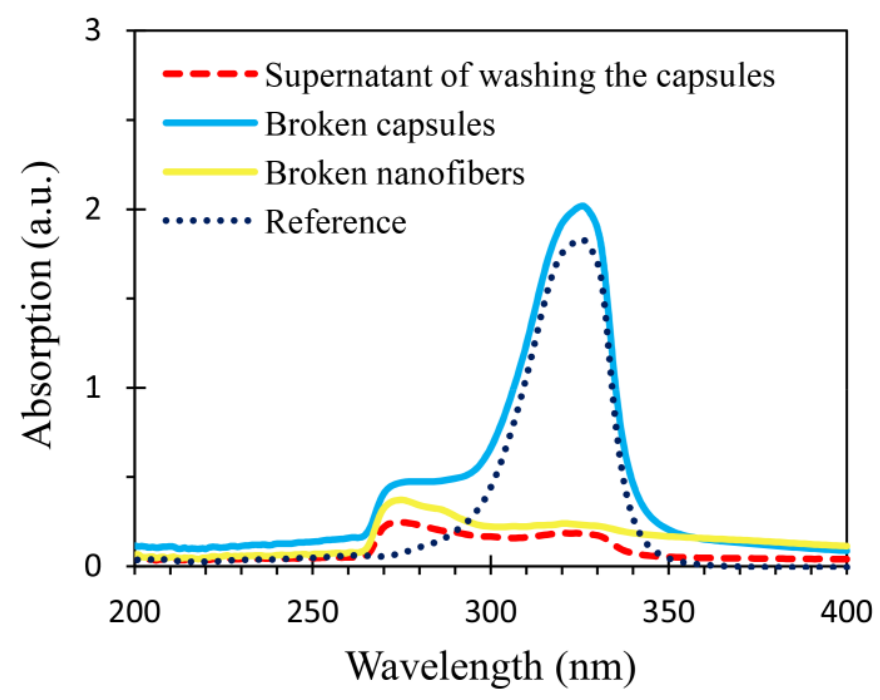

Figure 3. UV-vis spectra of the supernatants of 3 times of washing of the capsules (red dashed line), broken microspheres (blue line) and nanofibers (yellow line) of polyaniline after the same MBT encapsulation procedure, and the reference spectrum related to direct dissolution of MBT in ethanol (dark blue dotted line). 

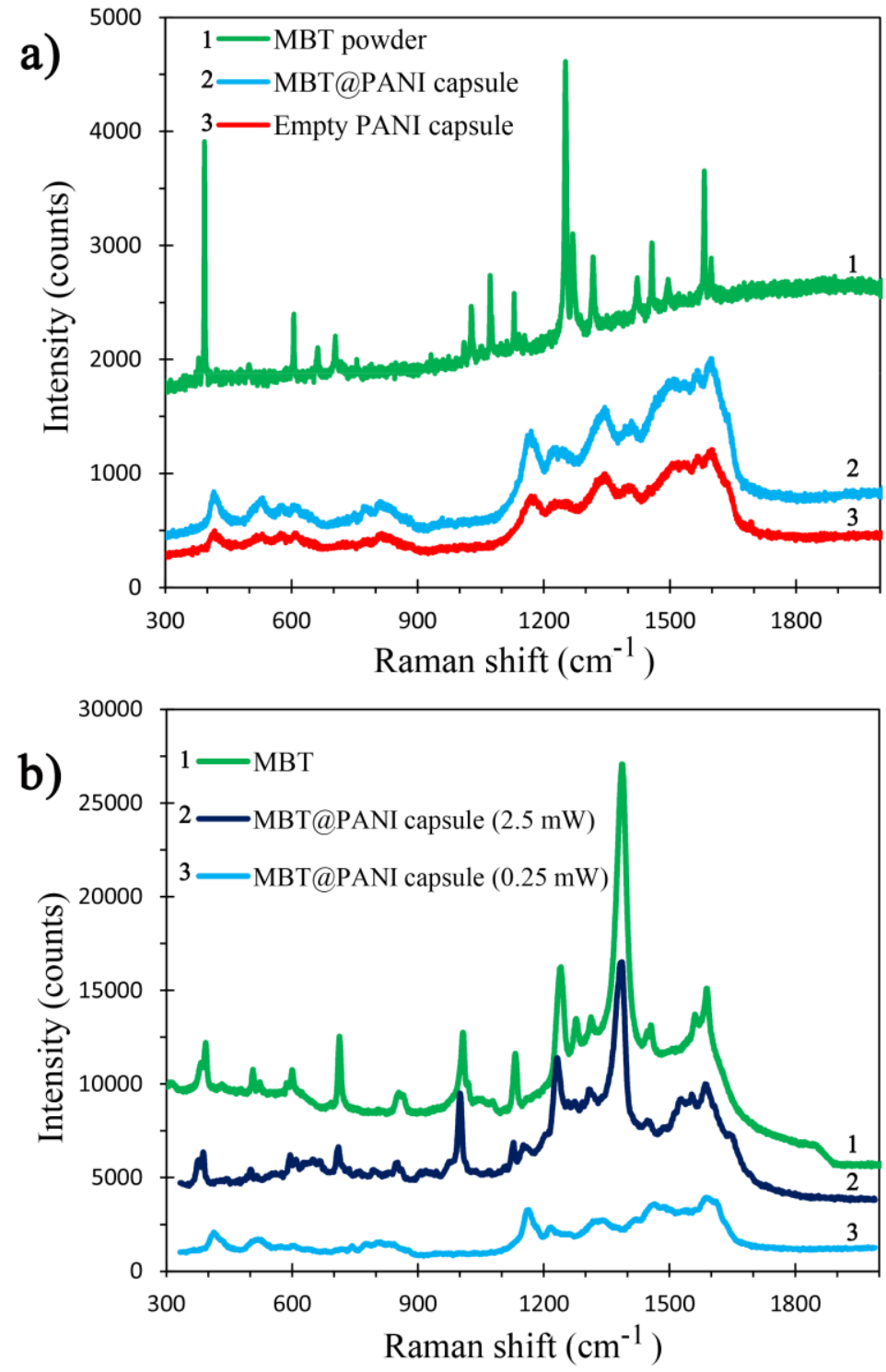

Figure 4. (a) Raman spectra of MBT Powder, MBT loaded PANI capsule and empty PANI capsule. (b) SERS spectra of MBT and MBT loaded capsules in water; the capsules were first studied with lower laser power $(0.25 \mathrm{~mW})$ which resulted in the same spectra as the hollow capsules, and then 10 times higher laser power $(2.5 \mathrm{~mW})$ was applied, which caused the capsules to burn off and MBT was released and absorbed on the SERS probe. 


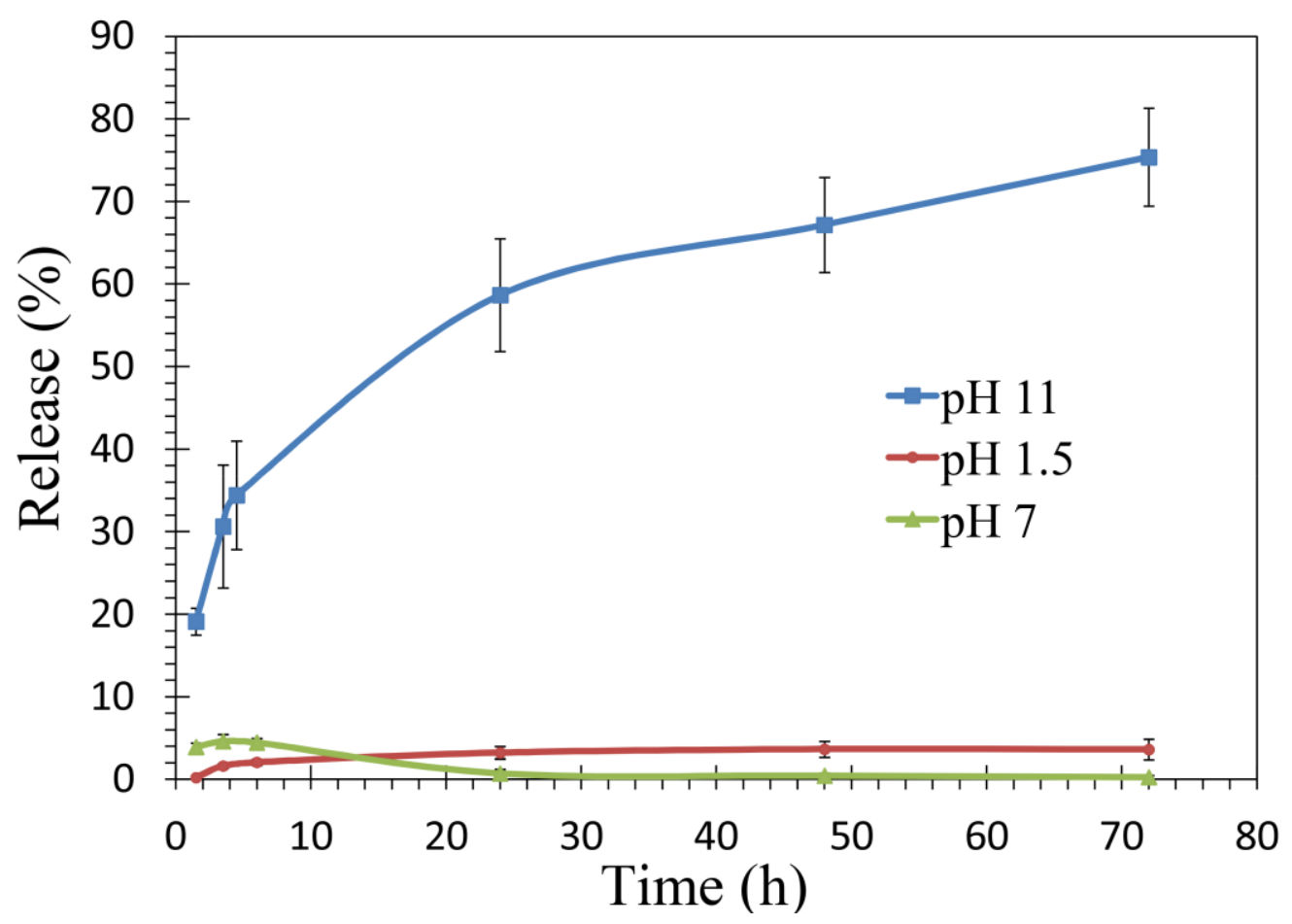

Figure 5. Release profile of MBT from polyaniline capsules at different pHs. 


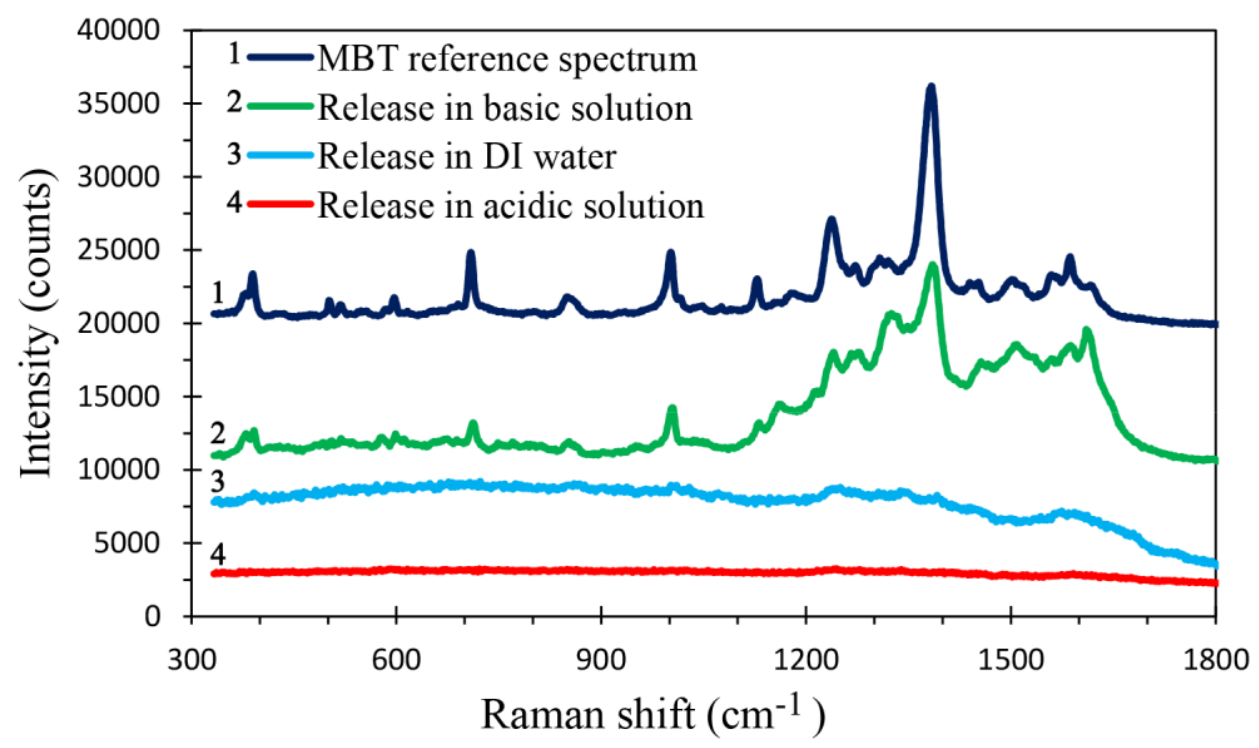

Figure 6. SERS spectra of release of MBT from PANI capsules at different pHs; MBT reference spectrum is the spectrum of MBT powder directly dissolved in basic solution. 


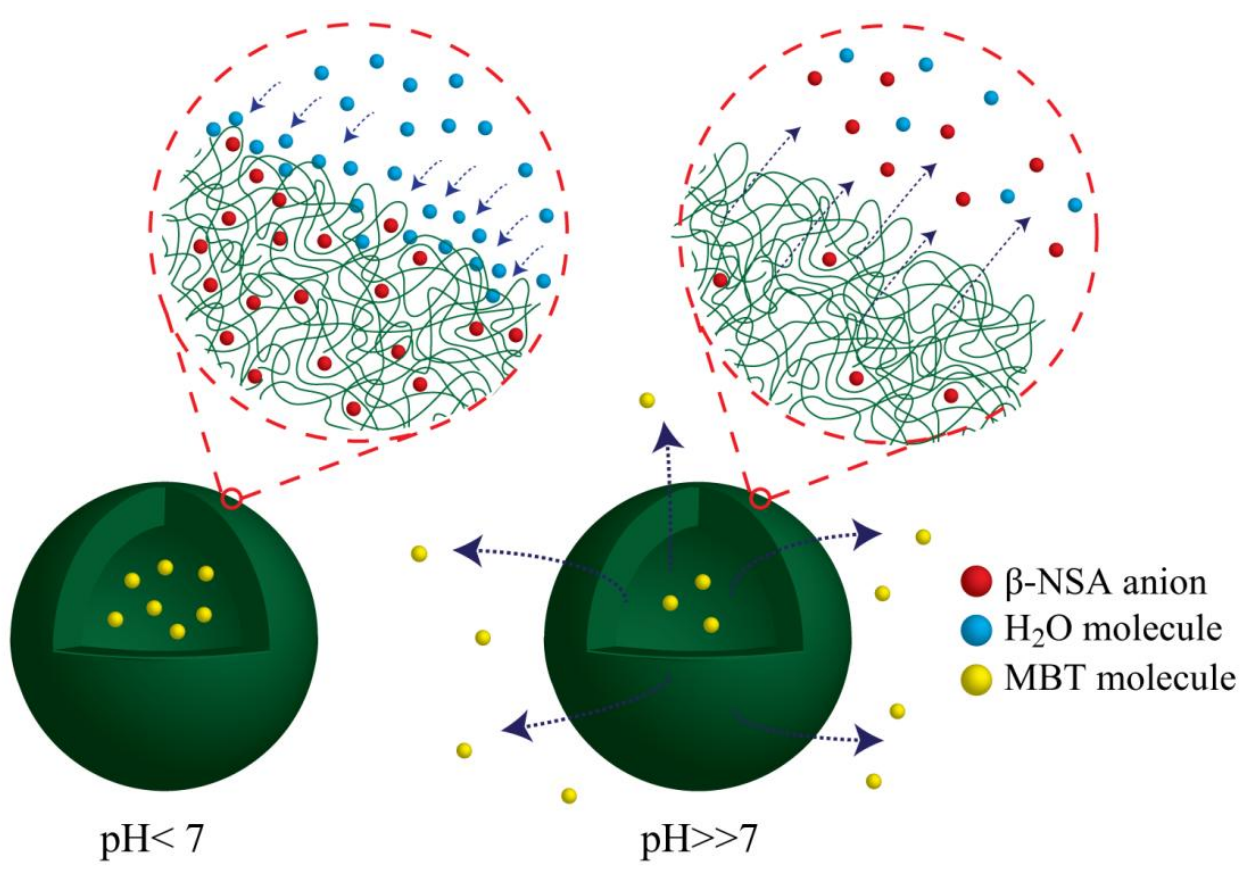

Figure 7. Schematic representation of the mechanism of $\mathrm{pH}$ responsive release of $\mathrm{MBT}$ from PANI capsules. 

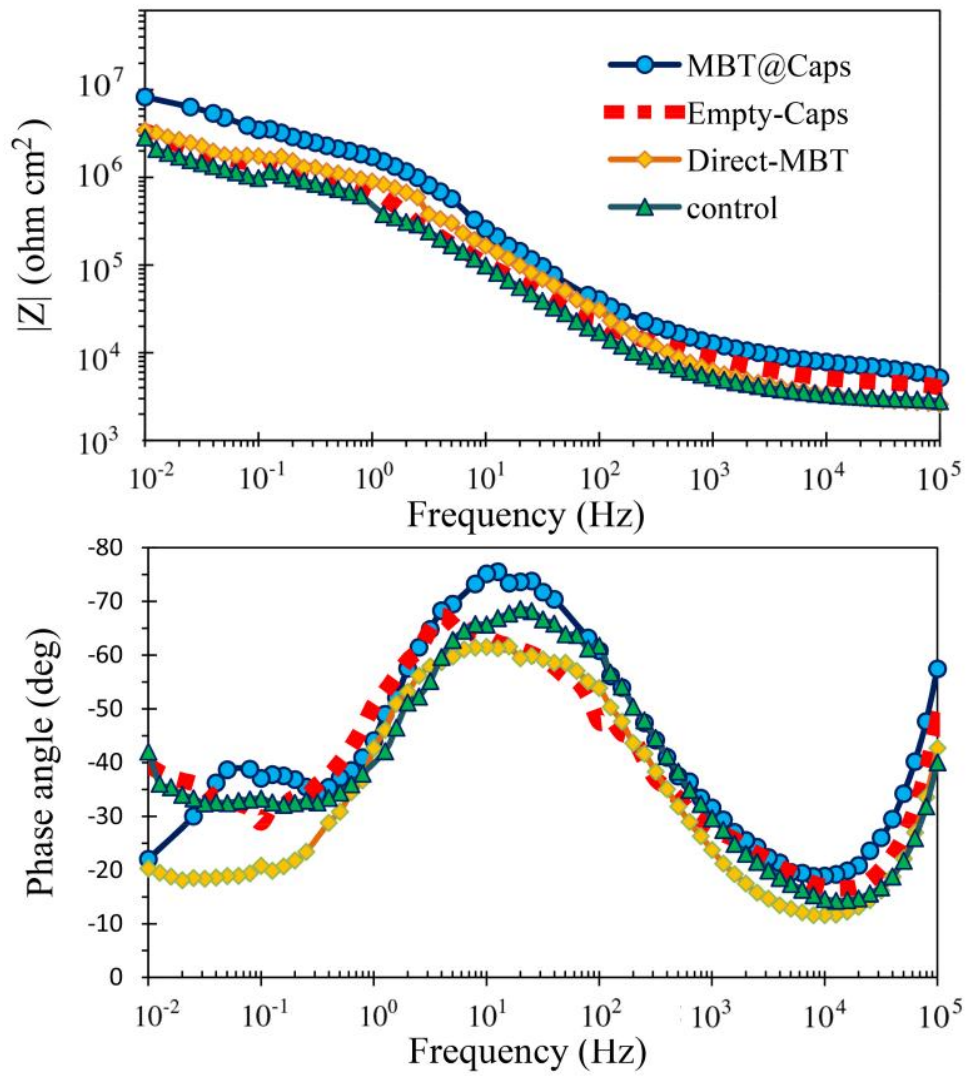

Figure 8. Bode plots of the scratched samples after 1 day immersion in $0.3 \mathrm{wt} \% \mathrm{NaCl}$ solution. 

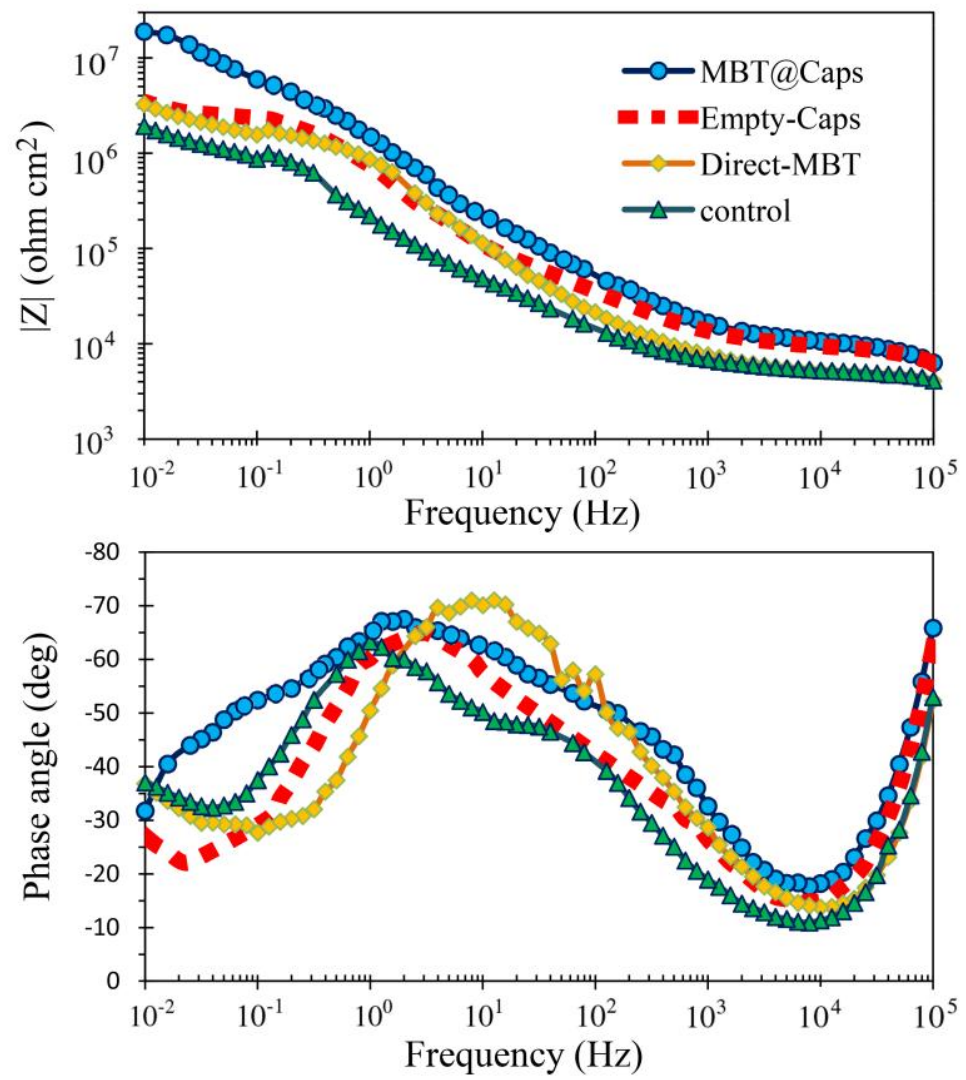

Figure 9. Bode plots of the scratched samples after 7 days immersion in $0.3 \mathrm{wt} \% \mathrm{NaCl}$ solution. 
a)

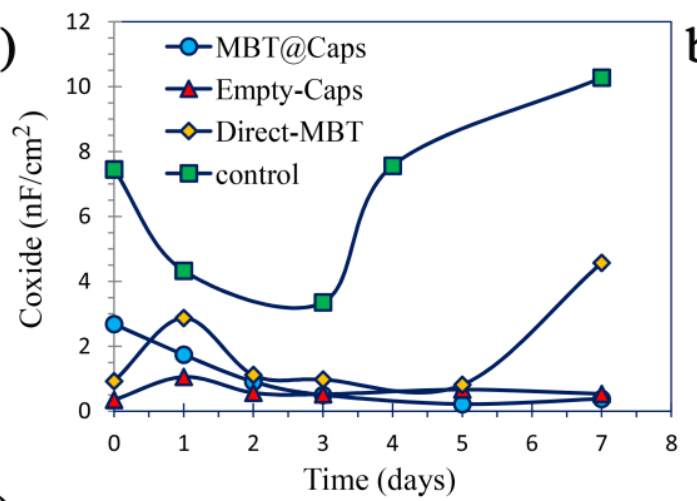

c)

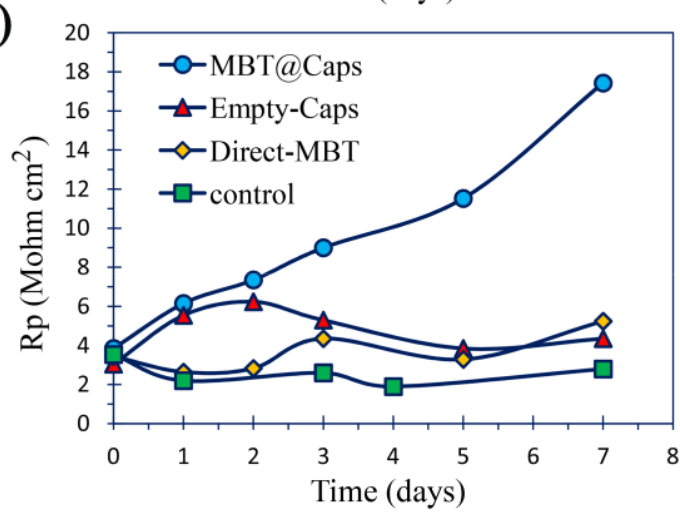

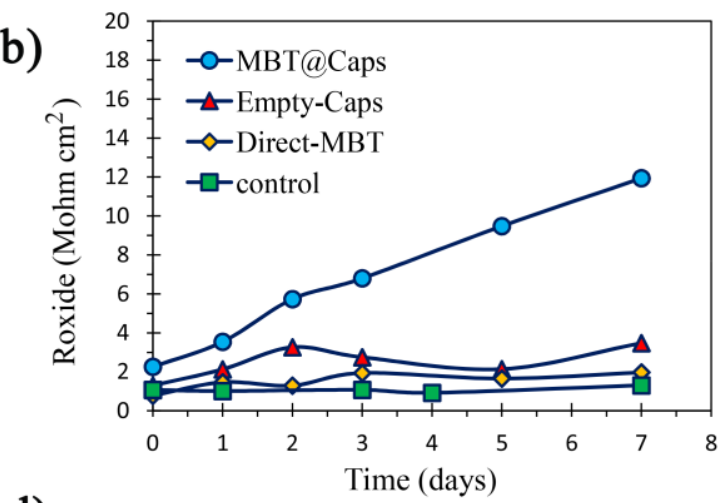

d)

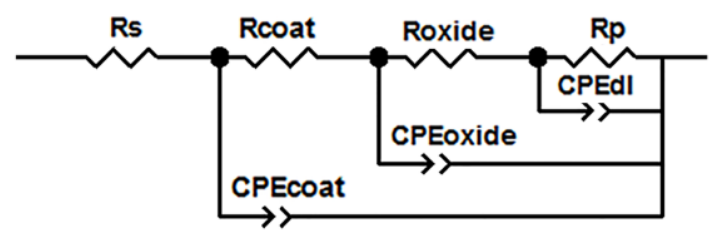

Figure 10. Evolution of (a) capacitance and (b) resistance of oxide layer; and (c) polarization resistance, for different coating systems during immersion; and (d) the equivalent circuit used for numerical fittings. 

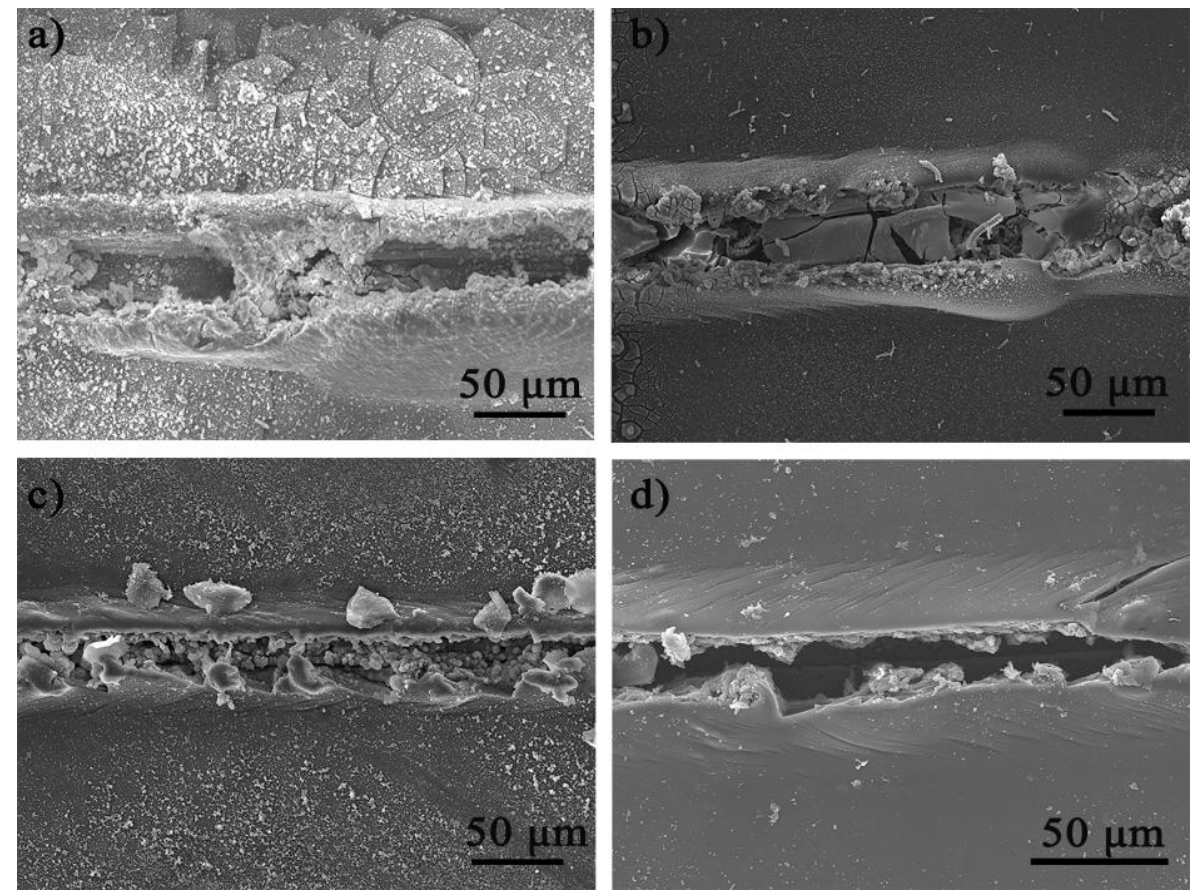

Figure 11. SEM images of the scratched coating systems: (a) control, (b) Direct-MBT, (c) Empty-Caps, and (d) MBT@Caps; after 4 days of immersion in 0.3 wt\% NaCl solution. 


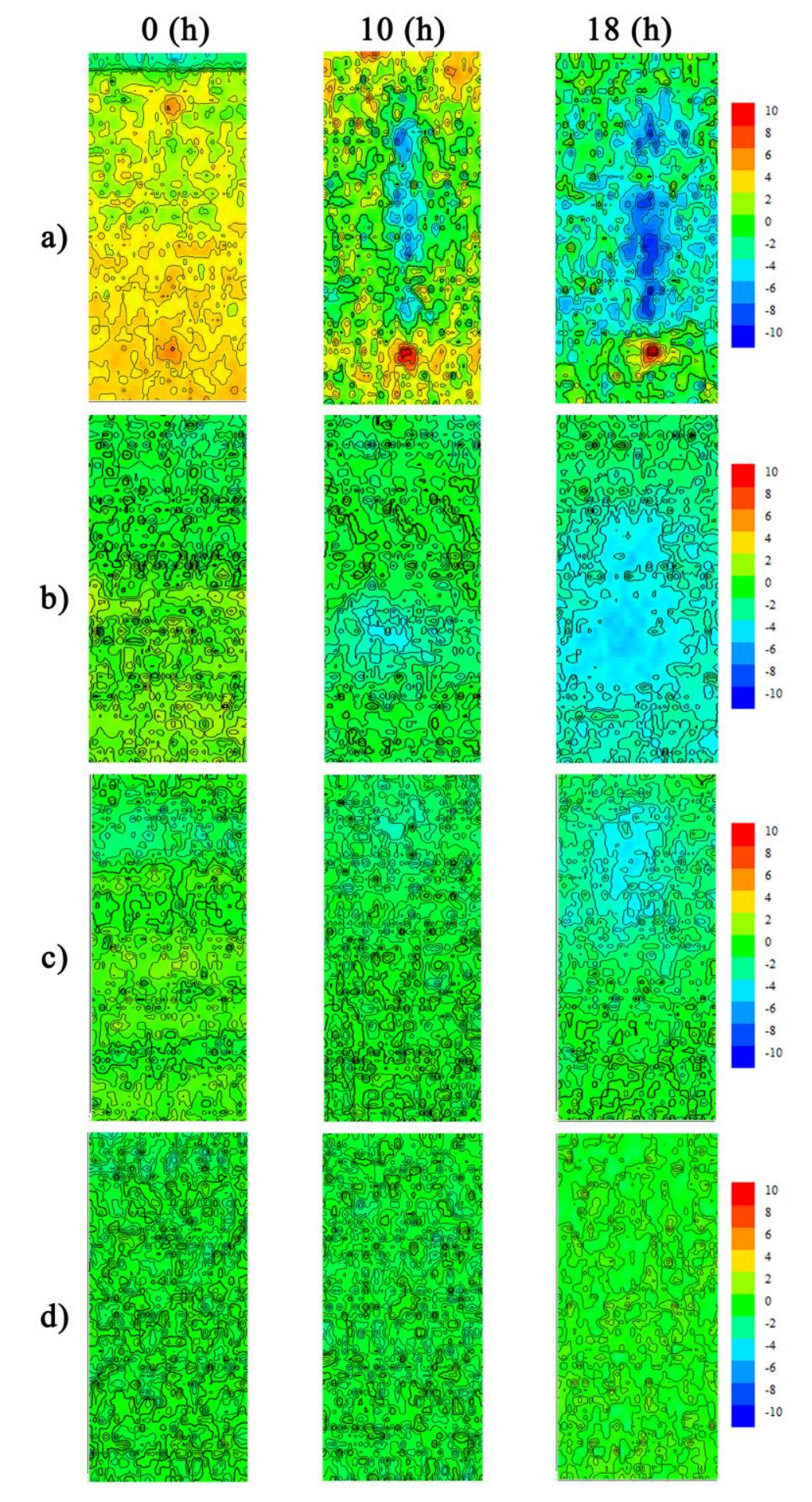

Figure 12. SVET current density maps of a scratch on: (a) control, (b) Direct-MBT, (c)

Empty-Caps, and (d) MBT@Caps coating systems after exposure to 0.3 wt\% $\mathrm{NaCl}$ solution for 0,10 and $18 \mathrm{~h}$. Scale units: $\mu$ A.cm ${ }^{-2}$. Scanned area: $\approx 1 \mathrm{~mm} \times 2 \mathrm{~mm}$. 

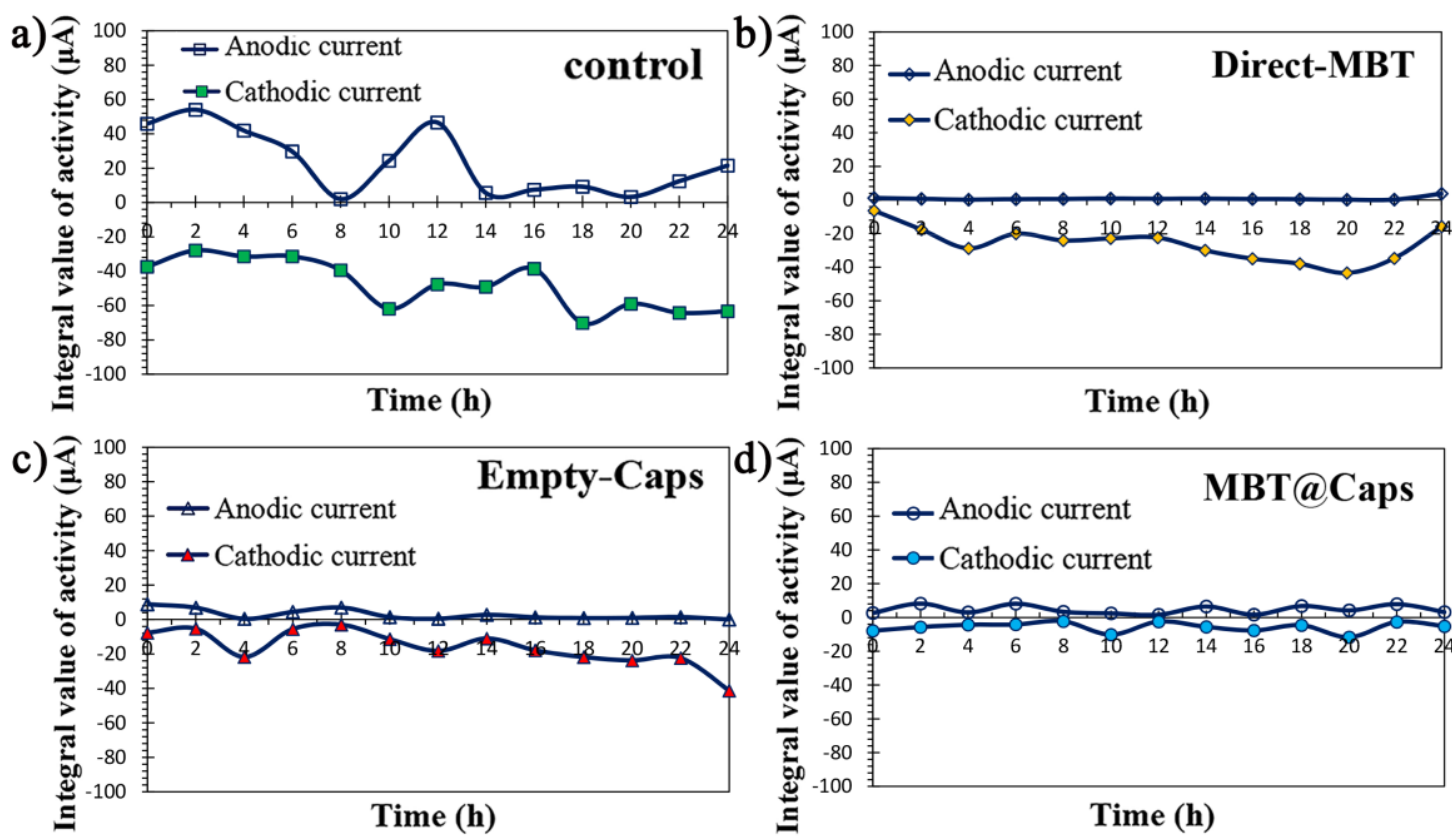

Figure 13. Evolution with time of the total anodic and cathodic currents from SVET

measurements for the four different coating systems: (a) control, (b) Direct-MBT, (c) EmptyCaps, and (d) MBT@Caps. 

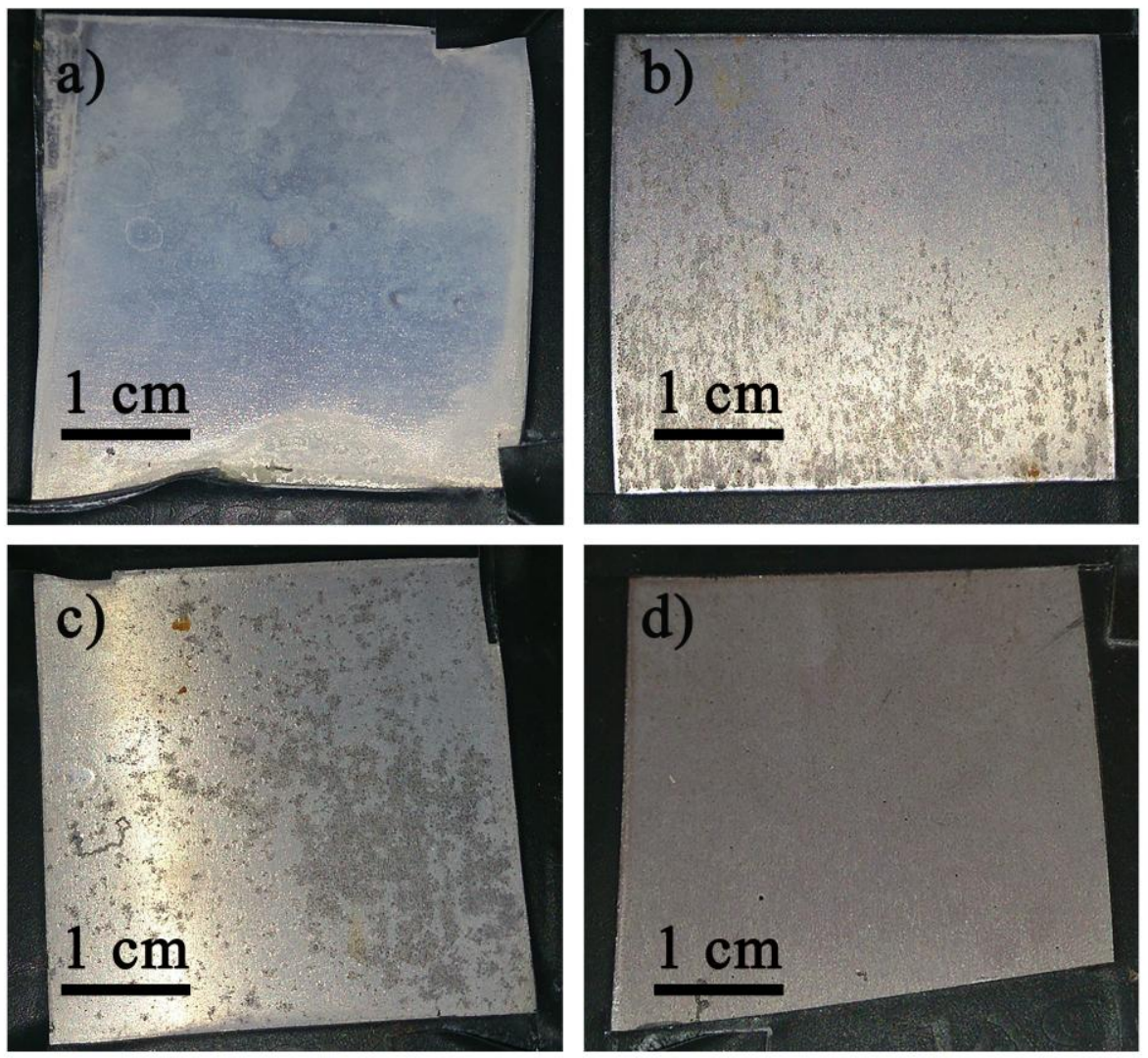

Figure 14. Visual appearance of AA2024 samples coated with different coating systems: (a) control, (b)Direct-MBT, (c) Empty-Caps, and (d) MBT@Caps; after 7 months of exposure to salt spray test. 